TITLE:

\title{
Modified Faraday rotation in a three-dimensional magnetophotonic opal crystal consisting of maghemite/silica composite spheres
}

\section{$\operatorname{AUTHOR}(\mathrm{S})$ :}

Murai, Shunsuke; Yao, Situ; Nakamura, Tadashi; Kawamoto, Takahiro; Fujita, Koji; Yano, Kazuhisa; Tanaka, Katsuhisa

\section{CITATION:}

Murai, Shunsuke ... [et al]. Modified Faraday rotation in a three-dimensional magnetophotonic opal crystal consisting of maghemite/silica composite spheres. Applied Physics Letters 2012, 101(15): 151121.

ISSUE DATE:

2012-10

URL:

http://hdl.handle.net/2433/160697

RIGHT:

(C) 2012 American Institute of Physics 


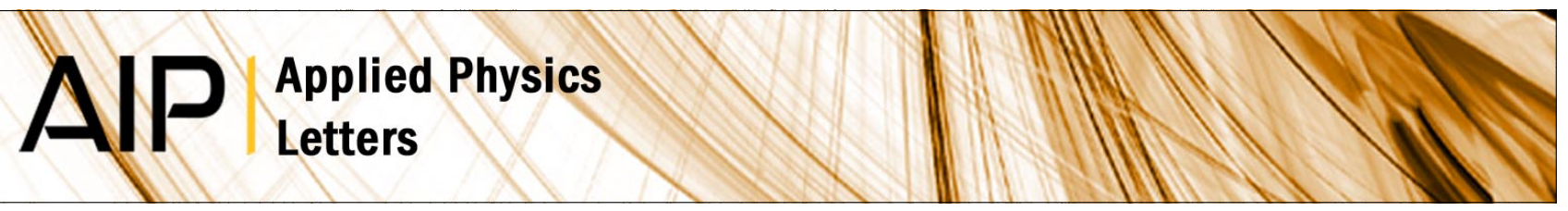

\section{Modified Faraday rotation in a three-dimensional magnetophotonic opal crystal consisting of maghemite/silica composite spheres}

Shunsuke Murai, Situ Yao, Tadashi Nakamura, Takahiro Kawamoto, Koji Fujita et al.

Citation: Appl. Phys. Lett. 101, 151121 (2012); doi: 10.1063/1.4757608

View online: http://dx.doi.org/10.1063/1.4757608

View Table of Contents: http://apl.aip.org/resource/1/APPLAB/v101/i15

Published by the American Institute of Physics.

\section{Related Articles}

Diamondoid coating enables disruptive approach for chemical and magnetic imaging with $10 \mathrm{~nm}$ spatial resolution Appl. Phys. Lett. 101, 163101 (2012)

Assembled $\mathrm{Fe} 3 \mathrm{O} 4$ nanoparticles on graphene for enhanced electromagnetic wave losses Appl. Phys. Lett. 101, 153108 (2012)

Highly versatile ultra-low density GaAs quantum dots fabricated by filling of self-assembled nanoholes Appl. Phys. Lett. 101, 143106 (2012)

Superstructure of self-aligned hexagonal GaN nanorods formed on nitrided $\mathrm{Si}(111)$ surface Appl. Phys. Lett. 101, 131605 (2012)

Self-assembled NaNbO3-Nb2O5 (ferroelectric-semiconductor) heterostructures grown on LaAIO3 substrates Appl. Phys. Lett. 101, 132902 (2012)

\section{Additional information on Appl. Phys. Lett.}

Journal Homepage: http://apl.aip.org/

Journal Information: http://apl.aip.org/about/about_the_journal

Top downloads: http://apl.aip.org/features/most_downloaded

Information for Authors: http://apl.aip.org/authors

\section{ADVERTISEMENT}
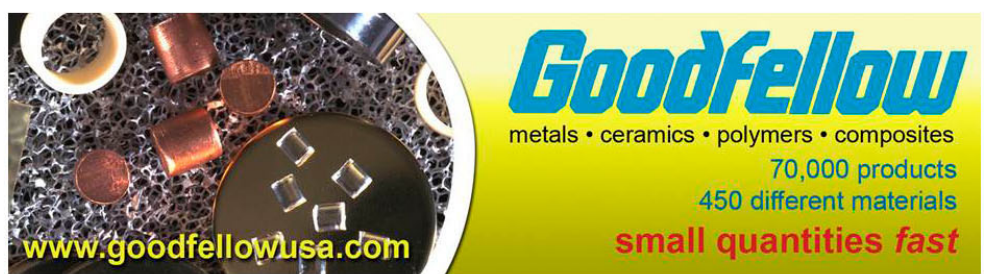


\title{
Modified Faraday rotation in a three-dimensional magnetophotonic opal crystal consisting of maghemite/silica composite spheres
}

\author{
Shunsuke Murai, ${ }^{1, a)}$ Situ Yao, ${ }^{1}$ Tadashi Nakamura, ${ }^{2}$ Takahiro Kawamoto, ${ }^{1}$ Koji Fujita, ${ }^{1}$ \\ Kazuhisa Yano, ${ }^{2}$ and Katsuhisa Tanaka ${ }^{1}$ \\ ${ }^{1}$ Department of Material Chemistry, Graduate School of Engineering, Kyoto University, Katsura, Nishikyo-ku, \\ Kyoto 615-8510, Japan \\ ${ }^{2}$ Toyota Central R \& D Labs., Inc., 41-1 Yokomichi, Nagakute, Aichi 480-1192, Japan
}

(Received 6 August 2012; accepted 18 September 2012; published online 11 October 2012)

\begin{abstract}
We have prepared a three-dimensional magnetophotonic crystal and observed modulated Faraday rotation around its photonic band gap. The magnetophotonic crystal was prepared by the self-assembly of highly monodispersed silica spheres, which were heavily impregnated with maghemite $\left(\gamma-\mathrm{Fe}_{2} \mathrm{O}_{3}\right)$ nanoparticles. The slab sample with a thickness of $10 \mu \mathrm{m}$ shows a clear photonic band gap centered at $\lambda=620 \mathrm{~nm}$ in optical transmittance. Faraday rotation is notably modified inside the photonic band gap. (c) 2012 American Institute of Physics. [http://dx.doi.org/10.1063/1.4757608]
\end{abstract}

A photonic crystal (PC) is a periodic array of macroscopic dielectric materials of different refractive index on the scale of the optical wavelength. The presence of photonic band gap $(\mathrm{PBG})$, in which the propagation of electromagnetic waves is forbidden, is a well-known feature of PCs. ${ }^{1}$ This provides a mechanism to control and manipulate the flow of photons.

Constructing a PC from a magnetic material or filling a $\mathrm{PC}$ with a magnetic material can result in the formation of magnetophotonic crystals (MPCs). ${ }^{2}$ Such materials would give an extra degree of freedom to achieve enhanced nonreciprocal optical phenomena such as magneto-optical Faraday and Kerr effects. Indeed, it has been demonstrated that the magneto-optical response of one- (1D) $)^{3-8}$ and twodimensional (2D) MPCs is significantly enhanced at band edge frequencies. This remarkable result has prompted the investigation of three-dimensional (3D) MPCs in which the periodicity in the three directions could lead to a complete PBG in the optical regions.

Although achieving high-quality 3D MPCs is much more complex and still a challenge, there are several works producing a 3D MPC by using a self-assembly technique. ${ }^{9-15}$ The general process involves the self-assembly of monodispersed spheres into an fcc lattice, followed by an infiltration of magnetic materials in its interstices. A simple example is filling the interstices with a magnetic liquid, ${ }^{10}$ although in this case the MPC is not all-solid. In order to make all-solid 3D MPCs, Pavlov et al. infiltrated magnetite $\left(\mathrm{Fe}_{3} \mathrm{O}_{4}\right)$ nanoparticles into the self-assembled silica spheres. ${ }^{11}$ Recently, Caicedo et al. made a 3D inverse opal MPC consisting of Ni nanoparticles and alumina by using polystylene colloids as a sacrifice template. ${ }^{12}$ A general drawback of this approach, i.e., self-assembly and infiltration, is that although the self-assembled opal is highly ordered and periodic, the infiltration with magnetic nanoparticles inevitably disturbs the opal structure, making the PBG broader and less clear. This smears the effects the PBG on magnetooptical response of MPC. Actually, only a limited number

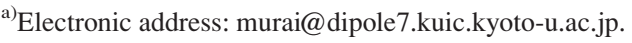

of 3D MPCs exhibit magneto-optical effects modified by PBG. $^{10-12}$

In this study, we present a building block by which an MPC can be built without being suffered from infiltration: Our MPC is constructed of highly monodispersed silica spheres inside which magnetic fine particles are heavily impregnated. Because they are inside the silica sphere, the presence of magnetic components does not directly disturb the self-assembly process. Here, our slab sample shows a clear dip due to PBG in the optical transmittance. The measurement of Faraday effect shows the modification of the rotation angle near the PBG.

The samples were prepared as follows: ${ }^{16}$ First, the monodispersed mesoporous silica sphere, MMSS, which possesses highly periodic mesopores, was prepared via a surfactant-templated sol-gel route. ${ }^{17,18}$ Then, ferrocenecarbaldehyde as an iron source was infiltrated in the mesopores and the silica spheres were heat treated at $1273 \mathrm{~K}$. During the heat treatment, maghemite $\left(\gamma-\mathrm{Fe}_{2} \mathrm{O}_{3}\right)$ nanoparticles were precipitated and grown to the size $\sim 3.1 \mathrm{~nm}$, while the mesopores were collapsed. The final spheres had an average diameter of $263 \mathrm{~nm}$ and contained as high as $21.2 \mathrm{wt} . \% \gamma-$ $\mathrm{Fe}_{2} \mathrm{O}_{3}$ measured by energy-dispersive x-ray spectroscopy (EDX). This amount is considerably higher than that used in the work by Fang et al., where $\mathrm{Fe}_{3} \mathrm{O}_{4}$ nanoparticles were loaded on silica spheres up to 6.4 wt. \%. ${ }^{15}$ Magnetic measurement proved that the spheres impregnated with $\gamma-$ $\mathrm{Fe}_{2} \mathrm{O}_{3}$ shows superparamagnetism at room temperature. ${ }^{16}$ Finally, by following the procedure described in Ref. 19, the $\gamma-\mathrm{Fe}_{2} \mathrm{O}_{3}$-impregnated spheres were assembled into a 3D MPC in between two slide glasses. In short, an aqueous dispersion of the $\gamma-\mathrm{Fe}_{2} \mathrm{O}_{3}$-impregnated spheres was injected into a fluidic cell, comprising two glass substrates (length: $38 \mathrm{~mm}$, width: $26 \mathrm{~mm}$, thickness: $0.7 \mathrm{~mm}$ ) and two spacers (thickness: $10 \mu \mathrm{m}$ ) sandwiched between them. The evaporation of water under ambient conditions resulted in the formation of an fcc opal with the (111) plane oriented parallel to the slide glass surface. A reference silica sample was also prepared following the same procedure by using the same MMSS heated at $1273 \mathrm{~K}$ but without an iron source inside. The resultant samples were dried in vacuo at $423 \mathrm{~K}$ prior to 
the measurements. The distance between adjacent layers in the [111] direction is $\sqrt[2]{2 / 3} \times D=215 \mathrm{~nm}$, where $D$ is the diameter of the sphere, so that the crystal with $10 \mu \mathrm{m}$ thick consists of $\sim 50$ layers.

The optical images of the sample are shown in Figs. 1(a) and 1(b), indicating that the sample has both glossy and matte domains. Scanning electron microscopic (SEM) images in Figs. 1(c) and 1(d) confirm that this comes from the difference in the spatial arrangement of spheres; the sample consists of ordered and disordered domains. It is also confirmed that no $\gamma-\mathrm{Fe}_{2} \mathrm{O}_{3}$ particle appears on the surface of silica spheres.

The optical transmittance was measured by using a spectrophotometer (JASCO, V-570). The Faraday rotation angles were measured at room temperature by the polarization modulation technique using a commercial measurement system (JASCO, Model K-250) with an Xe lamp as a light source. The measurements were also done for the pair of slide glasses in order to subtract the contribution of diamagnetic slide glasses sustaining the sample.

A problem in evaluating the magneto-optical response of opal-based MPCs is an optical activity that stems from their structures, which has been pointed out recently. ${ }^{12,14,20}$ Anisotropy appears both intrinsically due to the symmetry in opal structure and extrinsically due to internal strains during the fabrication. The anisotropy causes optical birefringence and dichroism, which overlap the magneto-optical response in the measurement. In order to separate those effects, we obtained the Faraday rotation data by subtracting the rotation data measured exactly at the same spot but without applying an external magnetic field from the raw data.

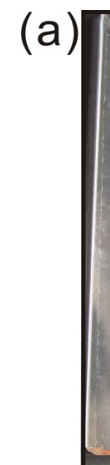

(c)
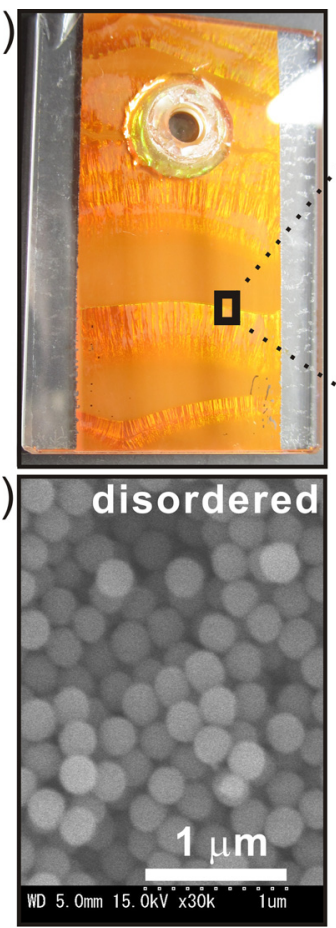

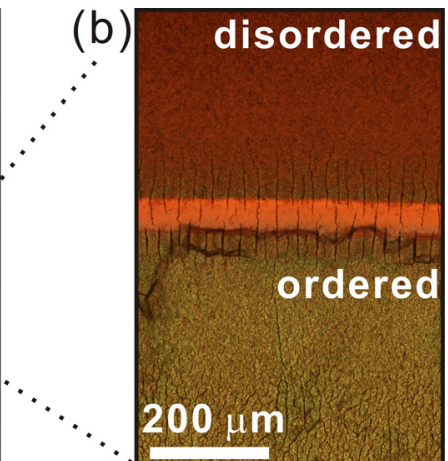

(d)

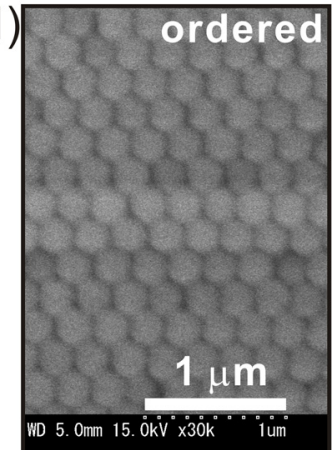

FIG. 1. (a) Optical image (top view) of the sample prepared in between two slide glasses. (b) Optical microscopic image of the sample, showing the interface between the ordered and disordered domains. (c) SEM image of the disordered domain of the sample. (d) SEM image of the ordered domain of the sample.
Figure 2(a) shows the optical transmittance $(T)$ of the sample, on both the ordered (denoted by a solid curve) and disordered (a dotted curve) domains. The data for the

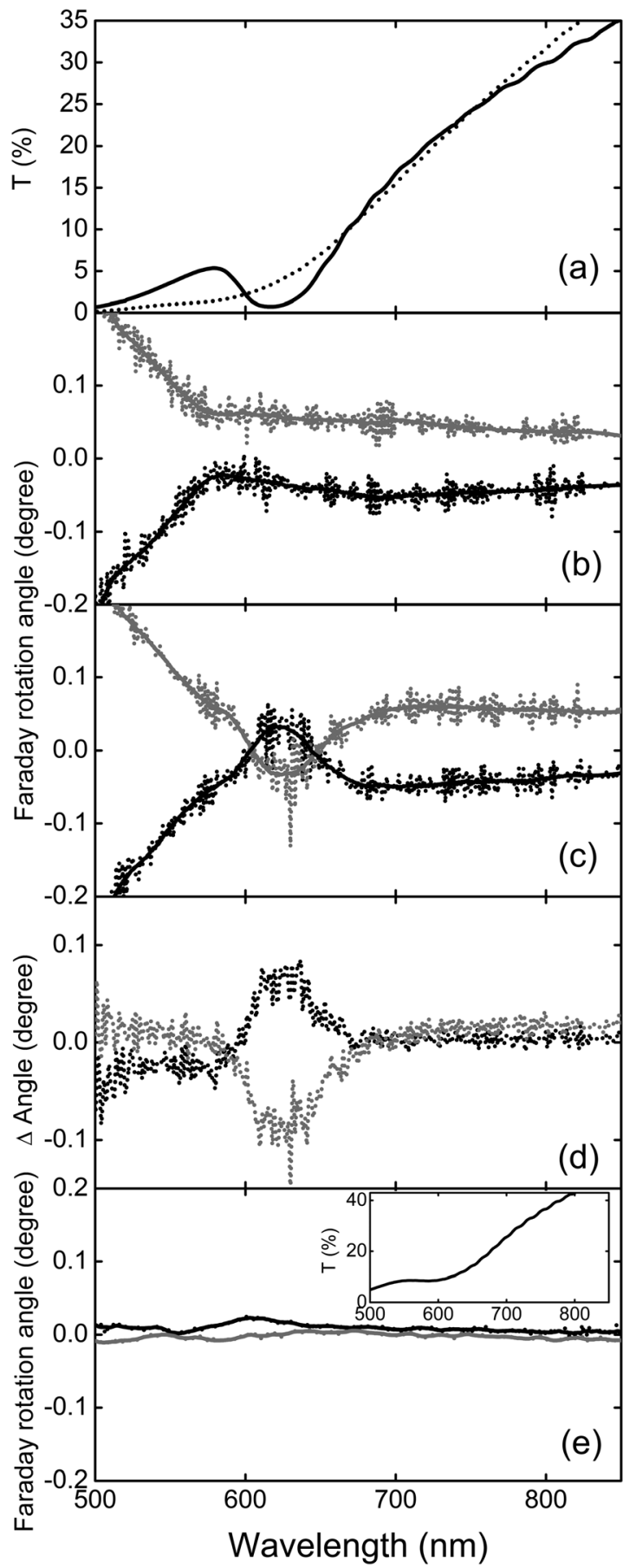

FIG. 2. (a) Optical transmittance of the disordered (a dotted curve) and ordered (a solid curve) domain of the sample. (b) and (c) Faraday rotation of the disordered (b) and ordered (c) domain of the sample. Curves are the guides for the eyes. The black and gray data denote the rotation with the external magnetic field of +15 and $-15 \mathrm{kOe}$, respectively. The data were plotted after subtracting the diamagnetic contribution from a pair of slide glasses sustaining the sample, and also the response of the sample without applying the magnetic field. (d) The difference between the Faraday rotation angle of the ordered domain and that of the disordered domain under the magnetic field of $+15 \mathrm{kOe}$ (black) and $-15 \mathrm{kOe}$ (gray). (e) Faraday rotation of the reference silica sample consisting of the same silica spheres but without $\gamma-\mathrm{Fe}_{2} \mathrm{O}_{3}$ inside: black $(+15 \mathrm{kOe})$, gray $(-15 \mathrm{kOe})$. The data were plotted after subtracting the diamagnetic contribution from a pair of slide glasses and the response of the reference sample under $0 \mathrm{kOe}$. The inset shows the optical transmittance of the reference sample. 
disordered domain show an absorption below $600 \mathrm{~nm}$, which comes from both an absorption of $\gamma-\mathrm{Fe}_{2} \mathrm{O}_{3}{ }^{21-23}$ and multiple light scattering by the spheres. While little difference is seen at longer wavelengths (typically $\lambda \geq 700 \mathrm{~nm}$ ), $T$ of the ordered domain shows a dip at around $\lambda=620 \mathrm{~nm}$, which corresponds to its PBG. It is also noticed that $T$ is larger for the ordered domain at the shorter wavelength edge of the PBG $(\lambda \leq 580 \mathrm{~nm})$.

Figures 2(b) and 2(c) show the Faraday rotation angle as a function of wavelength for the disordered and ordered domain, respectively. Here, the data taken without an external magnetic field, and the diamagnetic contribution from the pair of slide glasses, were subtracted from the raw data. The black and gray curves refer to the data taken by applying +15 and $-15 \mathrm{kOe}$, respectively. As shown in Figs. 2(b) and 2(c), these two curves are symmetric with respect to the $x$ axis (the wavelength axis), due to the nonreciprocal nature of the Faraday effect. This also indicates that the influence of structural optical activity, which is independent of the external magnetic field, was properly subtracted. The rotation observed in Fig. 2(b) is attributed to the superparamagnetic $\gamma-\mathrm{Fe}_{2} \mathrm{O}_{3}$ nanoparticles. ${ }^{21-24}$ The difference in refractive index $(\Delta n)$ for the left- and right-handed circularly polarized light is induced through the magnetic moments of individual nanoparticles oriented parallel to the external magnetic field. As a result, the polarization plane of the incident linearly polarized light is rotated as it travels inside the sample. Unlike the disordered domain shown in Fig. 2(b), the ordered domain exhibits notable modification around $\lambda=620 \mathrm{~nm}$ [Fig. 2(c)], corresponding to that of PBG in $T$ [Fig. 2(a)]. At this wavelength, the rotation angle is decreased in magnitude and changes its sign. This modification is better visualized in Fig. 2(d), where the Faraday rotation angle of the disordered domain was subtracted from that of the ordered one. The difference shows a maximum at $\lambda=625 \mathrm{~nm}$, which is in the middle of the PBG.

Figure 2(e) shows the Faraday rotation of the reference silica sample without $\gamma-\mathrm{Fe}_{2} \mathrm{O}_{3}$ nanoparticles. The sample exhibits a PBG in its transmission [see the inset of Fig. 2(e)], but no significant rotation is observed in the Faraday effect measurements. This indicates the small contribution of the silica spheres to the Faraday effect.

Figure 3 depicts the angle of Faraday rotation as a function of external magnetic field. In Fig. 3(a), we plot the data for the disordered domain at two wavelengths, $\lambda=560 \mathrm{~nm}$ (black) and $620 \mathrm{~nm}$ (gray). For the both wavelengths, the Faraday rotation shows a negative slope with respect to the magnetic field and is saturated at high magnetic fields with no hysteresis, confirming that the nanoparticles are superparamagnetic at room temperature. The slope of the curve is less steep for the data at $\lambda=620 \mathrm{~nm}$, which is in agreement with the observation in Fig. 2(b). Figure 3(b) shows the results for the ordered one. A black line indicates the data taken at $\lambda=560 \mathrm{~nm}$, i.e., outside the PBG, showing the same behavior as the disordered domain. In contrast, at $\lambda=620 \mathrm{~nm}$ (gray), inside the PBG, the behavior is similar to that of the disordered domain at lower magnetic fields, whereas at higher magnetic fields the slope changes its sign and becomes positive. This result indicates that the effect of PBG

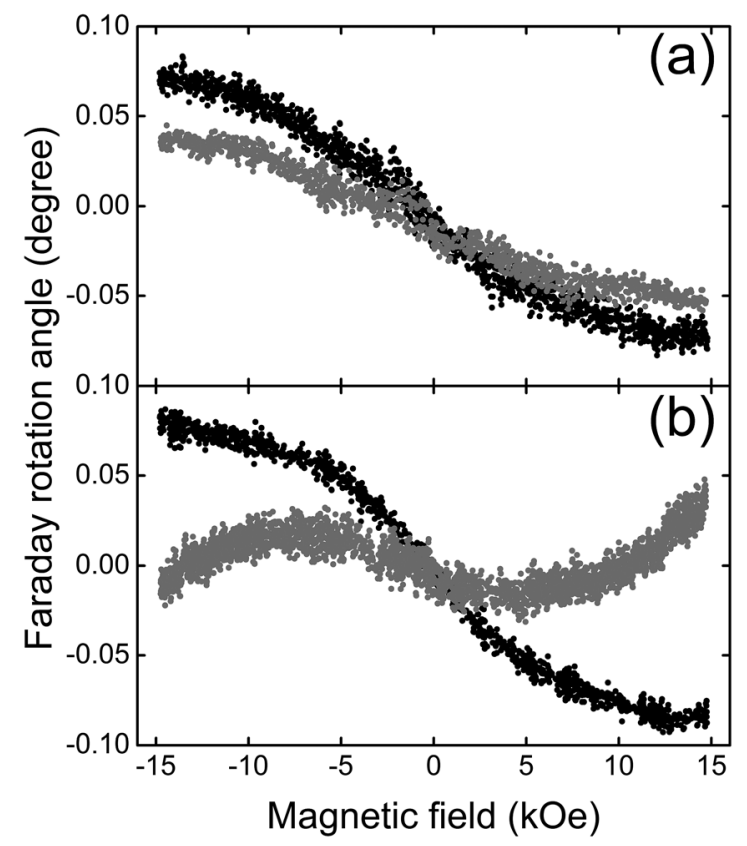

FIG. 3. Magnetic field dependence of the Faraday rotation for the disordered (a) and ordered (b) domain of the sample. The data were taken at $560 \mathrm{~nm}$ (black dots) and $620 \mathrm{~nm}$ (gray dots).

on the Faraday rotation is notable especially when the magnetic field is high.

The change in the Faraday rotation can be discussed in terms of the modification of effective refractive index by PBG. The presence of PBG causes an anomalous dispersion in refractive index associated with extinction by Bragg diffraction. $^{25,26}$ Since the condition of Bragg diffraction depends on the refractive index, a non-zero $\Delta n$ in PCs leads to a difference in the spectral position of PBG and thus a difference in the spectral range of anomalous dispersion for left- and right-handed circularly polarized light. Consequently, the $\Delta n$ induced by the magnetic field is further modulated around the spectral area of PBG, resulting in the modified Faraday rotation as seen in Fig. 2(c). This can also explain the observation in Fig. 3(b), where the modification is notable at higher magnetic fields. Preceding works on $1 \mathrm{D}^{8}$ and $3 \mathrm{D}^{12}$ MPCs highlighted the enhancement of the magneto-optical effect at the band edge due to a reduced group velocity, which is not very clear in our observation.

In summary, we have fabricated a magnetophotonic opal made of highly monodispersed silica spheres, heavily impregnated with $\gamma-\mathrm{Fe}_{2} \mathrm{O}_{3}$ nanoparticles. We examined its Faraday effect after subtracting the effect of optical activity that comes from its structure. The result shows that our material is one of the few 3D MPCs that exhibit a modified Faraday effect due to PBG, proving that the present technique, i.e., self-assembly of spheres heavily impregnated with magnetic nanoparticles, is effective in preparing 3D MPCs with good optical quality.

This study was supported by a Grant-in-Aid for Scientific Research (B, No. 24350104 and C, No. 24560824) and for Challenging Exploratory Research (No. 24656385) from the Ministry of Education, Culture, Sports, Science and Technology (MEXT), Japan. 
${ }^{1}$ K. Sakoda, Optical Properties of Photonic Crystals, 1st ed. (Springer-Verlag, Berlin, 2005).

${ }^{2}$ M. Inoue, R. Fujikawa, A. Baryshev, A. Khanikaev, P. B. Lim, H. Uchida, O. Aktsipetrov, A. Fedyanin, T. Murzina, and A. Granovsky, J. Phys. D: Appl. Phys. 39, R151 (2006).

${ }^{3}$ K. Takahashi, H. Takagi, K. H. Shin, H. Uchida, P. B. Lim, and M. Inoue, J. Appl. Phys. 101, 09C523 (2007).

${ }^{4}$ K. Takahashi, F. Kawanishi, S. Mito, H. Takagi, K. H. Shin, J. Kim, P. B. Lim, H. Uchida, and M. Inoue, J. Appl. Phys. 103, 07B331 (2008).

${ }^{5}$ J. Boriskina, S. Erokhin, A. Granovsky, A. Vinogradov, and M. Inoue, Phys. Solid State 48, 717 (2006).

${ }^{6}$ H. Kato, T. Matsushita, A. Takayama, M. Egawa, K. Nishimura, and M. Inoue, IEEE Trans. Magn. 38, 3246 (2002).

${ }^{7}$ M. Inoue, K. Arai, T. Fujii, and M. Abe, J. Appl. Phys. 83, 6768 (1998).

${ }^{8}$ A. Fedyanin, O. Aktsipetrov, D. Kobayashi, K. Nishimura, H. Uchida, and M. Inoue, J. Magn. Magn. Mater. 282, 256 (2004).

${ }^{9}$ A. V. Baryshev, T. Kodama, K. Nishimura, H. Uchida, and M. Inoue, J. Appl. Phys. 95, 7336 (2004).

${ }^{10}$ C. Koerdt, G. L. J. A. Rikken, and E. P. Petrov, Appl. Phys. Lett. 82, 1538 (2003).

${ }^{11}$ V. V. Pavlov, P. A. Usachev, R. V. Pisarev, D. A. Kurdyukov, S. F. Kaplan, A. V. Kimel, A. Kirilyuk, and T. Rasing, Appl. Phys. Lett. 93, 072502 (2008).

${ }^{12}$ J. M. Caicedo, O. Pascu, M. López-García, V. Canalejas, Á. Blanco, C. López, J. Fontcuberta, A. Roig, and G. Herranz, ACS Nano 5, 2957 (2011).
${ }^{13}$ J. M. Caicedo, E. Taboada, D. Hrabovský, M. López-García, G. Herranz, A. Roig, A. Blanco, C. López, and J. Fontcuberta, J. Magn. Magn. Mater. 322, 1494 (2010).

${ }^{14}$ I. Šimkienè, A. Rèza, A. Kindurys, V. Bukauskas, J. Babonas, R. Szymczak, P. Aleshkevych, M. Franckevičius, and R. Vaišnoras, Lith. J. Phys. 50, 7 (2010).

${ }^{15}$ M. Fang, T. T. Volotinen, S. K. Kulkarni, L. Belova, and K. V. Rao, J. Appl. Phys. 108, 103501 (2010).

${ }^{16}$ T. Nakamura, Y. Yamada, and K. Yano, J. Mater. Chem. 16, 2417 (2006).

${ }^{17}$ K. Yano and Y. Fukushima, J. Mater. Chem. 14, 1579 (2004).

${ }^{18}$ T. Nakamura, H. Yamada, Y. Yamada, A. Gurtanyel, S. Hartmann, N. Husing, and K. Yano, Langmuir 26, 2002 (2010).

${ }^{19}$ M. Ishii, H. Nakamura, H. Nakano, A. Tsukigase, and M. Harada, Langmuir 21, 5367 (2005).

${ }^{20}$ A. Rèza, I. Šimkienė, R. Vaišnoras, C. López, D. Golmayo, and J. Babonas, Photonics Nanostruct. Fundam. Appl. 9, 82 (2011).

${ }^{21}$ H. Wang, J. Shen, and J. Qian, J. Magn. Magn. Mater. 73, 103 (1988).

${ }^{22}$ P. K. Jain, Y. Xiao, R. Walsworth, and A. E. Cohen, Nano Lett. 9, 1644 (2009).

${ }^{23}$ T. Tepper, C. Ross, and G. Dionne, IEEE Trans. Magn. 40, 1685 (2004).

${ }^{24}$ Z. Šimša, P. Thailhades, L. Presmanes, and C. Bonningue, J. Magn. Magn. Mater. 242-245(Part 1), 381 (2002).

${ }^{25}$ J. F. Galisteo-López, M. Galli, M. Patrini, A. Balestreri, L. C. Andreani, and C. López, Phys. Rev. B 73, 125103 (2006).

${ }^{26}$ M. Centini, C. Sibilia, M. Scalora, G. D'Aguanno, M. Bertolotti, M. J. Bloemer, C. M. Bowden, and I. Nefedov, Phys. Rev. E 60, 4891 (1999). 